Article

\title{
Linear Maps that Preserve Any Two Term Ranks on Matrix Spaces over Anti-Negative Semirings
}

\author{
Kyung Tae Kang ${ }^{1}$, Seok-Zun Song $1, * \mathbb{C}$ and Young Bae Jun ${ }^{2}$ \\ 1 Department of Mathematics, Jeju National University, Jeju 63243, Korea; ktkang@jejunu.ac.kr \\ 2 Department of Mathematics Education, Gyeongsang National University, Jinju 52828, Korea; \\ skywine@gmail.com \\ * Correspondence: szsong@jejunu.ac.kr
}

Received: 30 October 2019; Accepted: 20 December 2019; Published: 1 January 2020

\begin{abstract}
There are many characterizations of linear operators from various matrix spaces into themselves which preserve term rank. In this research, we characterize the linear maps which preserve any two term ranks between different matrix spaces over anti-negative semirings, which extends the previous results on characterizations of linear operators from some matrix spaces into themselves. That is, a linear map $T$ from $p \times q$ matrix spaces into $m \times n$ matrix spaces preserves any two term ranks if and only if $T$ preserves all term ranks if and only if $T$ is a $(P, Q, B)$-block map.
\end{abstract}

Keywords: matrix space; anti-negative semiring; term rank; linear map; $(P, Q, B)$-block map

MSC: 15A03; 15A04; 15A86

\section{Introduction}

There are many characterizations of linear operators from various matrix spaces into themselves which preserve term rank. Beasley and Pullman ([1]) determined the linear operators on the $p \times q$ Boolean matrices, which preserve Boolean term rank. Their results are the following: For a linear operator on the $p \times q$ Boolean matrices,

$T$ preserves Boolean term rank if and only if it preserves Boolean term ranks 1 and 2;

$T$ preserves Boolean term rank if and only if it doubly preserves Boolean term rank 1 or $p$.

Beasley et al. ([2]) characterized linear operators on the $p \times q$ matrices over a commutative anti-negative semiring which preserve term rank. The results are the following: For a linear operator on the $p \times q$ commutative anti-negative semiring matrices,

$T$ preserves term rank if and only if it preserves term ranks 1 and $k$.

Song and Beasley([3]) characterized the linear maps that preserve term rank between different Boolean matrix spaces.

For the case of symmetric matrices, we have some results on the term rank preservers in [4,5].

In this paper, we investigate the characterizations of linear maps which preserve term rank between different matrix spaces over anti-negative commutative semirings, which extends the previous results on characterizations of linear operators between different matrix spaces.

\section{Preliminaries}

In this section, we give some definitions and basic results for our main results. 
A semiring [1] is a set $\mathbb{S}$ with addition $(+)$ and multiplication $(\cdot)$ such that $(\mathbb{S},+)$ is a commutative semigroup with identity 0 and $(\mathbb{S}, \cdot)$ is a semigroup with identity 1 . Moreover, the operation $\cdot$ is distributive over + . A semiring is called anti-negative if only 0 has an additive inverse. A semiring is called commutative if $s \cdot t=t \cdot s$ for all $s, t \in \mathbb{S}$.

The following are interesting examples of some anti-negative commutative semirings.

For a fixed positive integer $h$, let $\mathbb{B}_{h}$ be the Boolean algebra [6] of subsets of an $h$-element set $\mathbb{S}_{h}$ and $\sigma_{1}, \sigma_{2}, \ldots, \sigma_{h}$ denote the singleton subsets of $\mathbb{S}_{h}$. Union is denoted by + (addition) and intersection by juxtaposition (multiplication); 0 denotes the empty set and 1 the whole set $\mathbb{S}_{h}$. Under these two operations, $\mathbb{B}_{h}$ is a commutative anti-negative semiring, which is called generalized Boolean algebra. Then all of its elements, except 0 and 1 , are zero-divisors because each proper subset of $\mathbb{S}_{h}$ has empty intersection with its complement. In particular, if $h=1, \mathbb{B}_{1}$ is called the binary Boolean algebra [6].

Let $\mathbb{C}$ be any set with at least two elements. If $\mathbb{C}$ is totally ordered by $<$ (i.e., $a<b$ or $b<a$ for any two distinct elements $a, b$ in $\mathbb{C})$, then define $a+b$ as $\max (a, b)$ and $a b$ as $\min (a, b)$ for all $a, b \in \mathbb{C}$. If $\mathbb{C}$ has a universal upper bound and a universal lower bound, then $\mathbb{C}$ is a semiring, which is called a chain semiring [6].

Let $\mathbb{R}$ be the field of real numbers, let $\mathbb{F}=\{a \in \mathbb{R} \mid 0 \leq a \leq 1\}$ be a subset of $\mathbb{R}$. Define $a+b=$ $\max \{a, b\}$ and $a \cdot b=\min \{a, b\}$ for any $a, b \in \mathbb{F}$. Thus $(\mathbb{F},+, \cdot)$ is a chain semiring, which is called a fuzzy semiring [7]. In particular, if we take $\mathbb{F}=\{r\}$, a singleton set and we denote $\varnothing$ by 0 and $\{r\}$ by 1 , then this chain semiring is the binary Boolean algebra $\mathbb{B}=\{0,1\}$, which is a subsemiring of any chain semiring. Since a Boolean algebra $\mathbb{B}_{k}(k \geq 2)$ is not totally ordered under inclusion, it does not become a chain semiring.

In the following, $\mathbb{S}$ will denote an arbitrary commutative anti-negative semiring. For all $\mathrm{a}, \mathrm{b}$ $\in \mathbb{S}$, we supress the dot of $\mathrm{a} \cdot \mathrm{b}$, and simply write $\mathrm{ab}$. Let $\mathbb{M}_{p, q}(\mathbb{S})\left(\mathbb{M}_{m, n}(\mathbb{S})\right)$ be the set of all $p \times q$ $\left(m \times n\right.$ respectively) matrices with entries in $\mathbb{S}$. The addition and multiplication on $\mathbb{M}_{p, q}(\mathbb{S})$ are defined as usual.

In the following, we assume that integers $\mathrm{p}, \mathrm{q}, \mathrm{m}$ and $\mathrm{n}$ are positive integers with $p \leq q$ and $m \leq n$.

Let $E_{h, k}$ be the $p \times q(0,1)$-matrix whose only $(h, k)$ th entry is 1 , and $E_{h, k}$ be called a cell. $O$ is the $p \times q$ zero matrix, $I_{p}$ is the $p \times p$ identity matrix and $J$ is the $p \times q$ matrix all of whose entries are 1 .

A $p \times q$ matrix $R_{h}=\sum_{k=1}^{q} E_{h, k}$ is the $h$ th full row matrix and $C_{k}=\sum_{h=1}^{p} E_{h, k}$ is the $k$ th full column matrix. A $p \times q$ matrix $L$ is called a full line matrix if $L=R_{h}$ or $L=C_{k}$ for some $h \in\{1, \ldots, p\}$ or for some $k \in\{1, \ldots, q\}$.

A line of matrix $U \in \mathbb{M}_{p, q}(\mathbb{S})$ is a column or a row of $U$.

A matrix $U \in \mathbb{M}_{p, q}(\mathbb{S})$ has TR $\mathrm{t}$ (or term rank $\mathrm{t}$ ) if the minimum number of lines needed to cover all nonzero entries of $U$ is equal to $t$. We denote $\tau(U)=t$ if $U$ has TR $t$.

For matrices $U, V \in \mathbb{M}_{p, q}(\mathbb{S})$, it is said that $V=\left[v_{i, j}\right]$ dominates $U=\left[u_{i, j}\right]$ (denoted $U \sqsubseteq V$ or $V \sqsupseteq U)$ if $v_{h, k}=0$ implies $u_{h, k}=0$ for all $h$ and $k$.

Lemma 1. For matrices $U, V \in \mathbb{M}_{p, q}(\mathbb{S})$, we have

(1) $\tau(U+V) \leq \tau(U)+\tau(V)$;

(2) $\tau(U) \leq \tau(U+V)$;

(3) $U \sqsubseteq V$ implies that $\tau(U) \leq \tau(V)$.

Proof. (1) It is trivial from the definition.

(2) If $\tau(U)=t$, then we can find $t$ lines that cover all nonzero entries of $U$. If these lines cover all nonzero entries of $V$, then $\tau(U)=\tau(U+V)$. However, If these lines cannot cover all nonzero entries of $V$, then $\tau(U)<\tau(U+V)$. Thus, $\tau(U) \leq \tau(U+V)$.

(3) If $\tau(V)=t$, then we can find $t$ lines that cover all nonzero entries of $V$. Since $U \sqsubseteq V$, these lines can cover all nonzero entries of $U$. Thus $\tau(U) \leq t=\tau(V)$. 
For a matrix $U$ and lists $i(C)$ and $i(R)$ of column and row indices, respectively, $U(i(R) \mid i(C))$ denotes the submatrix constructed by deleting the columns $i(C)$ and rows $i(R)$ from $U$ and $U[i(R) \mid$ $i(C)$ ] denotes the submatrix constructed by taking the columns $i(C)$ and rows $i(R)$ from $U$.

For matrices $U, V \in \mathbb{M}_{p, q}(\mathbb{S})$, the matrix $U \circ V$ denotes the Schur or Hadamard product of $U$ and $V$. That is, the $(h, k)^{t h}$ entry of $U \circ V$ is $u_{h, k} v_{h, k}$.

A map $T: \mathbb{M}_{p, q}(\mathbb{S}) \rightarrow \mathbb{M}_{m, n}(\mathbb{S})$ is said to be linear if $T(r U+s V)=r T(U)+s T(V)$ for all $r, s \in \mathbb{S}$ and $U, V \in \mathbb{M}_{p, q}(\mathbb{S})$.

If $T: \mathbb{M}_{p, q}(\mathbb{S}) \rightarrow \mathbb{M}_{m, n}(\mathbb{S})$ is a map, then $T$ is called a $(P, Q, B)$-block map if there is a $p \times p$ permutation matrix $P$ and a $q \times q$ permutation matrix $Q$, and $B \in \mathbb{M}_{p, q}(\mathbb{S})$ with nonzero $b_{h, k}$, such that

- $\quad p \leq m$ and $q \leq n$, and $T(U)=P[(U \circ B) \oplus O] Q$ for any $U \in \mathbb{M}_{p, q}(\mathbb{S})$ or

- $\quad p \leq n$ and $q \leq m$, and $T(U)=P\left[(U \circ B)^{t} \oplus O\right] Q$ for any $U \in \mathbb{M}_{p, q}(\mathbb{S})$.

It is obvious that $(P, Q, B)$-block map is a linear map.

\section{Linear Maps that Preserve TR of Matrices over Anti-Negative Commutative Semirings}

In this section, we characterize the linear maps that preserve TR of $p \times q$ matrices over anti-negative commutative semirings, which are contained in Theorem 2.

For a linear map $T: \mathbb{M}_{p, q}(\mathbb{S}) \rightarrow \mathbb{M}_{m, n}(\mathbb{S})$, we say that $T$

(i) preserves TR $t$ if $\tau(T(U))=t$ whenever $\tau(U)=t$ for all $U \in \mathbb{M}_{p, q}(\mathbb{S})$;

(ii) doubly (or strongly) preserves TR $t$ if $\tau(T(U))=t$ if and only if $\tau(U)=t$ for all $U \in \mathbb{M}_{p, q}(\mathbb{S})$;

(iii) preserves TR if it preserves any TR $t$ with $t \leq p$.

Throughout this section, $T$ denotes a linear map $T: \mathbb{M}_{p, q}(\mathbb{S}) \rightarrow \mathbb{M}_{m, n}(\mathbb{S})$.

Lemma 2. Let $T$ be a $(P, Q, B)$-block map. Then $T$ doubly preserves any $T R t$ for $t=1, \cdots, p$.

Suppose that $T$ is a $(P, Q, B)$-block map, and $U \in \mathbb{M}_{p, q}(\mathbb{S})$ with $\tau(U)=t$ with $t \leq p$. Then $T(U)=$ $P[(U \circ B) \oplus O] Q$ or $T(U)=P\left[(U \circ B)^{t} \oplus O\right] Q$.

Consider the first case: $T(U)=P[(U \circ B) \oplus O] Q$. Since all entries of $B$ are not zero, $\tau(U \circ B)=$ $\tau(U)$, and $\tau((U \circ B) \oplus O)=\tau(U \circ B)$. Since the permuting columns and rows does not change the TR, we have

$\tau(T(U))=\tau(P[(U \circ B) \oplus O] Q)=\tau((U \circ B) \oplus O)=\tau(U \circ B)=\tau(U)=t$.

Thus $T$ preserves TR $t$.

Conversely if $\tau(T(U))=t$, then

$\tau(T(U))=\tau(P[(U \circ B) \oplus O] Q)=\tau((U \circ B) \oplus O)=\tau(U \circ B)=\tau(U)$.

Thus $\tau(U)=t$. That is, T doubly preserves TR $t$.

Consider the second case: $T(U)=P\left[(U \circ B)^{t} \oplus O\right] Q$. As in the first case, a similar argument shows the same results. That is, $T$ doubly preserves TR $t$.

Theorem 1. Let $T$ doubly preserve $T R 1$. Then $T$ is a $(P, Q, B)$-block map, and vice versa. (Here, we have either $m \geq p$ and $n \geq q$, or $n \geq p$ and $m \geq q$.)

Suppose that $T$ doubly preserves TR 1 . Then, the image of each line under $T$ in $\mathbb{M}_{p, q}(\mathbb{S})$ is a line in $\mathbb{M}_{m, n}(\mathbb{S})$ since $T$ preserves TR 1 . Thus we assume that either $T\left(R_{1}\right) \sqsubseteq R_{1}$ or $T\left(R_{1}\right) \sqsubseteq C_{1}$.

Consider the first case: $T\left(R_{1}\right) \sqsubseteq R_{1}$. Assume that $T\left(C_{k}\right) \sqsubseteq R_{h}$. Then, since $E_{1, k}$ is in both $R_{1}$ and $C_{k}$ and since $T\left(E_{1, k}\right) \neq O$ and lies in the first row $R_{1}, h$ must be 1 . However, then, for $k \neq j T\left(E_{1, j}+E_{2, k}\right) \sqsubseteq$ $R_{1}$. Hence, $T\left(E_{1, j}+E_{2, k}\right)$ has TR 1 . However, $\tau\left(E_{1, j}+E_{2, k}\right)=2$, a contradiction. Thus we conclude that the image of any full column matrix is contained in a column matrix. By a similar argument, the image of any full row matrix is contained in a row matrix. And, since two columns have TR 2 , the image of distinct full columns must be contained in distinct columns. Let $\alpha:\{1, \cdots p\} \rightarrow\{1, \cdots, m\}$ be 
defined by $\alpha(h)=k$ if $T\left(R_{h}\right) \sqsubseteq R_{k}$ and define $\beta:\{1, \cdots q\} \rightarrow\{1, \cdots, n\}$ by $\beta(h)=k$ if $T\left(C_{h}\right) \sqsubseteq C_{k}$. Then, $\alpha$ and $\beta$ are injective maps, and hence, $p \leq m$ and $q \leq n$. Let $\alpha^{\prime}:\{1, \cdots, m\} \rightarrow\{1, \cdots, m\}$ and $\beta^{\prime}:\{1, \cdots, n\} \rightarrow\{1, \cdots, n\}$ be a bijective maps such that $\left.\alpha^{\prime}\right|_{\{1, \cdots, p\}}=\alpha$ and $\left.\beta^{\prime}\right|_{\{1, \cdots, q\}}=\beta$. Let $P_{\alpha^{\prime}}$ and $Q_{\beta^{\prime}}$ be the permutation matrices of order $\mathrm{p}$ and $\mathrm{q}$, respectively, that correspond to the bijective maps $\alpha^{\prime}$ and $\beta^{\prime}$.

Thus we obtain that $p \leq m$ and $q \leq n$, and there is some nonzero $b_{h, k} \in \mathbb{S}$ such that $B=\left[b_{h, k}\right]$, $T\left(E_{h, k}\right)=b_{h, k}\left(P_{\alpha^{\prime}}\left[E_{r, s} \oplus O\right] Q_{\beta^{\prime}}\right)$ for every cell $E_{h, k}$. Therefore,

$$
\begin{aligned}
T(U) & =T\left(\sum_{h=1}^{p} \sum_{k=1}^{q} u_{h, k} E_{h, k}\right)=\sum_{h=1}^{p} \sum_{k=1}^{q} u_{h, k} T\left(E_{h, k}\right) \\
& =\sum_{h=1}^{p} \sum_{k=1}^{q} u_{h, k} b_{h, k}\left(P\left[E_{h, k} \oplus O\right] Q\right)=P[(U \circ B) \oplus O] Q
\end{aligned}
$$

for every $U=\left[u_{i, j}\right] \in \mathbb{M}_{p, q}(\mathbb{S})$. Thus $T$ is a $(P, Q, B)$-block map.

Consider the second case: $T\left(R_{1}\right) \sqsubseteq C_{1}$. As in the first case, a similar argument implies that $p \leq n$ and $q \leq m$. We obtain $T\left(E_{h, k}\right)=b_{h, k}\left(P_{\alpha^{\prime}}\left[E_{h, k} \oplus O\right]^{t} Q_{\beta^{\prime}}\right)$ for all $E_{h, k}$. Hence

$$
\begin{aligned}
T(U) & =T\left(\sum_{h=1}^{p} \sum_{k=1}^{q} u_{h, k} E_{h, k}\right)=\sum_{h=1}^{p} \sum_{k=1}^{q} u_{h, k} T\left(E_{h, k}\right) \\
& =\sum_{h=1}^{p} \sum_{k=1}^{q} u_{h, k} b_{h, k}\left(P\left[E_{h, k} \oplus O\right]^{t} Q\right)=P\left[(U \circ B)^{t} \oplus O\right] Q
\end{aligned}
$$

for every $U=\left[u_{i, j}\right] \in \mathbb{M}_{p, q}(\mathbb{S})$. This implies that $T$ is a $(P, Q, B)$-block map.

Conversely, if $T$ is a $(P, Q, B)$-block map, then $T$ doubly preserves TR 1 by Lemma 2 .

Lemma 3. Suppose that $T$ preserves $T R 1$ and $T R t(\geq 2)$. Then we have

(1) $T$ doubly preserves TR 1 ;

(2) $T$ is a $(P, Q, B)$-block map.

(1) Consider the first case that $t=2$ : If $U \in \mathbb{M}_{p, q}(\mathbb{S})$ has TR 1 , then $T(U)$ also has TR 1.

Conversely, if $V \in \mathbb{M}_{p, q}(\mathbb{S})$ is the matrix with $\tau(T(V))=1$, and $\tau(V) \neq 1$, then $\tau(V) \geq 2$. However, $\tau(V) \neq 2$ since $\tau(V)=2$ implies $\tau(T(V))=2$ by assumption that $t=2$. Thus $\tau(V) \geq 3$. Let $V_{1} \sqsubseteq V$ such that $\tau\left(V_{1}\right)=2$ and $V=V_{1}+V_{2}$ with $\tau\left(V_{2}\right) \geq 1$. Then $T\left(V_{1}\right) \sqsubseteq T\left(V_{1}\right)+T\left(V_{2}\right)=$ $T\left(V_{1}+V_{2}\right)=T(V)$. Thus $2=\tau\left(T\left(V_{1}\right)\right) \leq \tau(T(V))=1$ by Lemma 1 , which inequality is impossible. That is, $T$ doubly preserves TR 1 .

Consider the second case that $t \geq 3$ : Assume that a TR 2 matrix is mapped to a TR 1 matrix. Then we may consider $\tau\left(T\left(E_{1,1}+E_{2,2}\right)\right)=1$ without loss of generality. Then, since $T$ preserves TR 1 and TR $t$,

$t=\tau\left(T\left(E_{1,1}+E_{2,2}+E_{3,3}+\cdots+E_{t, t}\right)\right)=\tau\left(T\left(E_{1,1}+E_{2,2}\right)+T\left(E_{3,3}\right)+\cdots+T\left(E_{t, t}\right)\right) \leq \tau\left(T\left(E_{1,1}+\right.\right.$ $\left.\left.\left.E_{2,2}\right)\right)+\tau\left(T\left(E_{3,3}\right)\right)+\cdots+\tau\left(T\left(E_{t, t}\right)\right)\right)=1+(t-2)<t$,

which is impossible. Hence, $T$ doubly preserves TR 1.

(2) By (1), $T$ doubly preserves TR 1 . Hence $T$ is a $(P, Q, B)$-block map by Theorem 1.

Lemma 4. Suppose that $T$ preserves $T R t$.

(1) If $t \geq 2$ and $T$ does not preserve $T R 1$, then there is a matrix $U$ such that $\tau(U)=1$ and $\tau(T(U)) \geq 2$.

(2) If $\tau(U) \leq t$ for some $U \in \mathbb{M}_{p, q}(\mathbb{S})$, then $\tau(T(U)) \leq t$.

(1) Assume that $T$ does not preserve TR 1 and $\tau(T(U)) \leq 1$ for all $U$ with $\tau(U)=1$. Then, there is a cell $E_{h, k}$ with $T\left(E_{h, k}\right)=O$. We may assume that $T\left(E_{1,1}\right)=O$ without loss of generality. Since $\tau\left(E_{1,1}+E_{2,2}+\cdots+E_{t, t}\right)=t$ and $T$ preserves TR $t$, we get $\tau\left(T\left(E_{2,2}+E_{3,3}+\cdots+E_{t, t}\right)\right)=$ 
$\tau\left(T\left(E_{1,1}+E_{2,2}+\cdots+E_{t, t}\right)\right)=t$. If we make $V=T\left(E_{2,2}+\cdots+E_{t, t}\right)$, then we can take some cells $C_{1}, C_{2}, \cdots, C_{t}$ such that $V \sqsupseteq C_{h}$ for all $h=1, \cdots, t$, and $\tau\left(C_{1}+C_{2}+\cdots+C_{t}\right)=t$. Since $T\left(E_{2,2}+\right.$ $\left.\cdots+E_{t, t}\right)=V \sqsupseteq C_{1}+C_{2}+\cdots+C_{t}$, there is a cell in $\left\{E_{2,2}, \cdots, E_{t, t}\right\}$ whose image dominates two cells in $\left\{C_{1}, C_{2}, \cdots, C_{t}\right\}$, which contradicts $\tau(T(U)) \leq 1$ for all $U$ with $\tau(U)=1$ in the assumption. This contradiction implies that there is a matrix $U$ such that $\tau(U)=1$ and $\tau(T(U)) \geq 2$.

(2) If $\tau(U)=t$, then $\tau(T(U))=t$ since $T$ preserves TR $t$. Assume that $\tau(U)=s<t$, and $\tau(T(U))>t$. Then there exists a matrix $V$ such that $\tau(U+V)=t$ and hence $\tau(T(U+V))=t$. However, by Lemma 1 ,

$$
t=\tau(T(U+V))=\tau(T(U)+T(V)) \geq \tau(T(U))>t,
$$

which is impossible. Therefore it follows that $\tau(T(U)) \leq t$.

Lemma 5. If $T$ preserves any $T R t(\geq 2)$, but does not preserve $T R 1$, then $\tau(T(J)) \leq(t+2)$, where $J$ is the $p \times q$ matrix with all entries 1 .

By Lemma 4, if $T$ does not preserve TR 1 , then there is some matrix $U$ such that $\tau(U)=1$ and $\tau(T(U)) \geq 2$. So without loss of generality we may assume that $T\left(E_{1,1}+E_{1,2}\right) \sqsupseteq E_{1,1}+E_{2,2}$.

Assume that $\tau(T(J)) \geq(t+3)$. Then, $\tau(T(J)[3, \cdots, m \mid 3, \cdots, n]) \geq(t-1)$. So we may assume that $T(J)[3, \cdots, m \mid 3, \cdots, n] \sqsupseteq E_{3,3}+E_{4,4}+\cdots+E_{t+1, t+1}$ without loss of generality. Thus, there are $(t-1)$ cells, $C_{3}, C_{4}, \cdots, C_{t+1}$ such that $T\left(C_{3}+C_{4}+\cdots+C_{t+1}\right) \sqsupseteq E_{3,3}+E_{4,4}+\cdots+E_{t+1, t+1}$. Then, $T\left(E_{1,1}+E_{1,2}+C_{3}+C_{4}+\cdots+C_{t+1}\right) \sqsupseteq I_{t+1}=E_{1,1}+E_{2,2}+\cdots+E_{t+1, t+1}$. However, $\tau\left(E_{1,1}+E_{1,2}+\right.$ $\left.C_{3}+C_{4}+\cdots+C_{t+1}\right) \leq t$ and $\tau\left(T\left(E_{1,1}+E_{1,2}+C_{3}+C_{4}+\cdots+C_{t+1}\right)\right) \geq(t+1)$, which contradicts Lemma 4 (2). Thus, $\tau(T(J)) \leq(t+2)$.

Lemma 6. Let $i, j>t$. If $\tau\left(E_{1,1}+\cdots+E_{t, t}+U\right) \geq(t+1)$ and $U[t+1, \cdots, i \mid t+1, \cdots, j]=O$, then there is some $h, 1 \leq h \leq t$, such that $\tau\left(E_{1,1}+\cdots+E_{h-1, h-1}+E_{h+1 h+1}+\cdots+E_{t, t}+U\right) \geq(t+1)$.

Assume that $V=E_{1,1}+\cdots+E_{t, t}+U$ and $\tau(V) \geq(t+1)$. Then there are $(t+1)$ cells $C_{1}, C_{2}, \cdots, C_{t+1}$ such that $V \sqsupseteq C_{1}+C_{2}+\cdots+C_{t+1}$ and $\tau\left(C_{1}+C_{2}+\cdots+C_{t+1}\right)=(t+1)$. If $C_{1}+C_{2}+\cdots+C_{t+1} \sqsupseteq I_{t} \oplus O$ then some cell $C_{k}$ must be a cell $E_{a, b}$ where $a, b \geq(t+1)$, which is in contradiction with the assumption $U[t+1, \cdots, i \mid t+1, \cdots, j]=O$. Thus $C_{1}+C_{2}+\cdots+C_{t+1} \sqsupseteq I_{t} \oplus O$ does not hold. That is, there is some $h, 1 \leq h \leq t$, such that

$$
\tau\left(E_{1,1}+\cdots+E_{h-1, h-1}+E_{h+1, h+1}+\cdots+E_{t, t}+U\right) \geq(t+1) .
$$

Lemma 7. For $2 \leq(t+1)<p$, if $T$ preserves consecutive $T R t$ and $T R(t+1)$, then it preserves $T R 1$.

If $t=1$, we have finished. Assume that $t \geq 2$, and that $T$ does not preserve TR 1 . Then we have $\tau(T(J)) \leq(t+2)$ by Lemma 5. Since $T$ preserves TR $(t+1), \tau(T(J)) \geq(t+1)$.

Thus, $\tau(T(J))=(t+h)$ for $h=1$ or $h=2$. Now, we may assume that for some $i, j(<(t+h))$ with $(i+j)=(t+h), T(J)[i+1, \cdots, m \mid j+1, \cdots, n]=O$. This implies that

$$
T\left(E_{t+1, t+1}\right)[t+1, \cdots, m \mid t+1, \cdots, n]=O .
$$

Now, without loss of generality, we may assume that there are $(t+h)$ cells $C_{1}, C_{2}, \cdots, C_{t+h}$ such that $T\left(C_{r}\right) \sqsupseteq E_{r, r}$ for $r=1,2, \cdots,(t+h)$. Assume that the image of one cell in $\left\{C_{1}, C_{2}, \cdots, C_{t+h}\right\}$ dominates more than one cell in $\left\{E_{1,1}, E_{2,2}, \cdots, E_{t+1, t+1}\right\}$. Without loss of generality, we may assume that $T\left(C_{1}\right) \sqsupseteq E_{1,1}+E_{2,2}$. Then, $T\left(C_{1}+C_{3}+\cdots+C_{t+1}\right) \sqsupseteq E_{1,1}+E_{2,2}+\cdots+E_{t+1, t+1}$, which is a contradiction since $\tau\left(C_{1}+C_{3}+\cdots+C_{t+1}\right) \leq t$, and hence $\tau\left(T\left(C_{1}+C_{3}+\cdots+C_{k+1}\right)\right) \leq t$ by Lemma 4 (2), but $\tau\left(E_{1,1}+E_{2,2}+\cdots+E_{t+1, t+1}\right)=(t+1)$. Therefore, for each $r=1, \cdots,(t+1), T\left(C_{r}\right)$ dominates only one $E_{r, r}$. So, by permuting we may assume that $C_{1}+C_{2}+\cdots+C_{t} \sqsubseteq\left[\begin{array}{cc}J_{t} & O_{t, q-t} \\ O_{p-t, t} & O_{p-t, q-t}\end{array}\right]$. Consider $T\left(C_{1}+\cdots+C_{t}+E_{t+1, t+1}\right)=Z$. This matrix $Z$ must have TR $(t+1)$ and dominates 
$E_{1,1}+E_{2,2}+\cdots+E_{t, t}+T\left(E_{t+1, t+1}\right)$. Applying Equation (1) to Lemma 6, we can choose a cell $C_{s}$ in $\left\{C_{1}, \cdots, C_{t}\right\}$ such that $\tau\left(T\left(C_{1}+\cdots+C_{s-1}+C_{s+1}+\cdots+C_{t}+E_{t+1, t+1}\right)\right)=(t+1)$. However, $\tau\left(C_{1}+\cdots+C_{s-1}+C_{s+1}+\cdots+C_{t}+E_{t+1, t+1}\right)=t$, which is a contradiction.

This contradiction implies that $T$ preserve TR 1 .

Lemma 8. If $T$ preserves $T R$ t and $T R s$ with $p \geq s \geq(t+3)$, then it preserves $T R 1$.

Assume that $T$ does not preserve TR 1. Then $\tau(T(J)) \leq(t+2)$ by Lemma 5. For any $U \sqsubseteq J$, we have $\tau(T(U)) \leq \tau(T(J)) \leq t+2<s$ by Lemma 1 and assumption. However, if we take $U=I_{s}=E_{1,1}+E_{2,2}+\cdots+E_{s, s} \sqsubseteq J$, then $T\left(I_{s}\right)$ must have TR $s$ by assumption, while $\tau\left(T\left(I_{s}\right)\right) \leq$ $\tau(T(J)) \leq(t+2)<s$, a contradiction. That is, $\tau(T(U))=1$ for arbitrary TR 1 matrix $U$. Therefore $T$ preserves TR 1 .

Lemma 9. If $T$ preserves $T R t$ and $T R(t+2)$, then it preserves $T R(t+1)$.

Let $U \in \mathbb{M}_{p, q}(\mathbb{S})$.

First, consider the case that $\tau(U)=(t+1)$ and $\tau(T(U)) \geq(t+2)$. Let $U_{1}, U_{2}, \cdots, U_{t+1}$ be matrices of TR 1 such that $U=U_{1}+U_{2}+\cdots+U_{t+1}$. Since $\operatorname{tr}\left(T\left(U_{h}\right)\right) \geq 2$ for some $U_{h}$, we may assume that $\tau\left(T\left(U_{1}+U_{2}+\cdots+U_{h}\right)\right) \geq(h+1)$, for every $h=1,2, \cdots(t+1)$. However, then $\tau\left(U_{1}+\right.$ $\left.U_{2}+\cdots+U_{t}\right)=t$ while $\tau\left(T\left(U_{1}+U_{2}+\cdots+U_{t}\right)\right) \geq(t+1)$, a contradiction. Thus if $\tau(U)=(t+1)$, $\tau(T(U)) \leq(t+1)$.

Second, consider the cases $\tau(U)=(t+1)$ and $\tau(T(U))=r \leq t$. We may assume without loss of generality that $U=E_{1,1}+E_{2,2}+\cdots+E_{t+1, t+1}$ and $T(U) \sqsupseteq E_{1,1}+E_{2,2}+\cdots+E_{r, r}$. Then there are $r$ elements in $\left\{T\left(E_{1,1}\right), T\left(E_{2,2}\right), \cdots, T\left(E_{t+1, t+1}\right)\right\}$ whose sum dominates $E_{1,1}+E_{2,2}+\cdots+$ $E_{r, r}$. Say, without loss of generality, that $T\left(E_{1,1}+E_{2,2}+\cdots+E_{r, r}\right) \sqsupseteq E_{1,1}+E_{2,2}+\cdots+E_{r, r}$. Now, $\tau\left(U+E_{t+2, t+2}\right)=(t+2)$ so that $\tau\left(T\left(U+E_{t+2, t+2}\right)\right)=(t+2)$. However, since $\tau\left(T\left(U+E_{t+2, t+2}\right)\right)=$ $\tau\left(\left(T(U)+T\left(E_{t+2, t+2}\right)\right) \leq \tau(T(U))+\tau\left(T\left(E_{t+2, t+2}\right)\right)\right.$, it follows that $\tau\left(T\left(E_{t+2, t+2}\right)\right) \geq(t+2-r)$ and there are $r$ elements of $\left\{T\left(E_{1,1}\right), T\left(E_{2,2}\right), \cdots, T\left(E_{t+1, t+1}\right)\right\}$ whose sum with $T\left(E_{t+2, t+2}\right)$ has TR $(t+2)$, say $\tau\left(T\left(E_{1,1}+E_{2,2}+\cdots+E_{r, r}+E_{t+2, t+2}\right)\right)=(t+2)$. Since $r \leq t, \tau\left(E_{1,1}+E_{2,2}+\cdots+E_{r, r}+\right.$ $\left.E_{r+2, r+2}\right) \leq(r+1)$ and $\tau\left(T\left(E_{1,1}+E_{2,2}+\cdots+E_{r, r}+E_{t+2, t+2}\right)\right)=(t+2)$. By the above case, we have a contradiction.

Thus $T$ preserves TR $(t+1)$.

Lemma 10. If $T$ doubly preserves any one $T R t(\geq 2)$, then $T$ preserves $T R(t-1)$ and hence $T$ preserves $T R 1$.

Consider the first case $t=2$ : Assume that $U \in \mathbb{M}_{p, q}(\mathbb{S})$ has TR 1 . Then we may choose a matrix $V \in \mathbb{M}_{p, q}(\mathbb{S})$ such that $\tau(V)=1$ and $\tau(U+V)=2$. Since $\tau(T(U)) \leq \tau(T(U+V))=2$ since $T$ doubly preserves TR 2 . Since $\tau(T(U)) \neq 2$, it follows that $\tau(T(U))=1$. Thus the Lemma holds in the case $t=2$.

Consider the second case $t \geq 3$ : Let $U \in \mathbb{M}_{p, q}(\mathbb{S})$ and $\tau(U)=(t-1)$. Assume that $\tau(T(U))=$ $r<(t-1)$. Say we may assume that $\tau\left(T\left(E_{1,1}+\cdots+E_{t-1, t-1}\right)\right)=r<(t-1)$. Since $\tau\left(T\left(E_{1,1}+\right.\right.$ $\left.\left.\cdots+E_{t, t}\right)\right)=t$, it follows that $\tau\left(T\left(E_{t, t}\right)\right) \geq(t-r)$. So we may assume without loss of generality, that $T\left(E_{1,1}+\cdots+E_{t, t}\right) \sqsupseteq E_{1,1}+\cdots+E_{t, t}$ and that $T\left(E_{t, t}\right) \sqsupseteq E_{s+1, s+1}+\cdots+E_{t, t}$ for some $s \leq$ $r$. Thus, there are $s$ cells $\left\{E_{h_{1}, h_{1}}, \cdots, E_{h_{s}, h_{s}}\right\}$ in $\left\{E_{1,1}, \cdots, E_{t, t}\right\}$ such that $T\left(E_{h_{1}, h_{1}}+\cdots+E_{h_{s}, h_{s}}\right) \sqsupseteq$ $E_{1,1}+\cdots+E_{s, s}$. Then $T\left(E_{1,1}+\cdots+E_{t, t}\right) \sqsupseteq T\left(E_{h_{1}, h_{1}}+\cdots+E_{h_{s}, h_{s}}+E_{t, t}\right) \sqsupseteq E_{1,1}+\cdots+E_{t, t}$. Thus $\tau\left(T\left(E_{h_{1}, h_{1}}+\cdots+E_{h_{s}, h_{s}}+E_{t, t}\right)\right)=t$. However,

$$
\tau\left(E_{1,1}+\cdots+E_{s, s}+E_{t, t}\right)=(s+1) \leq(r+1)<(t-1)+1=t,
$$


which contradicts the assumption that $T$ doubly preserves TR $t$. Hence $\tau(T(U)) \geq(t-1)$. Further, $\tau(T(U)) \leq(t-1)$ by Lemma 4 (2), since $T$ doubly preserves TR $t$. Therefore $\tau(T(U))=(t-1)$, which implies that $T$ preserves TR $(t-1)$.

Moreover, $T$ preserves TR 1 by Lemma 7 .

Lemma 11. (1) If $T$ preserves any two $T R$ t and $T R s(>t)$, then $T$ is a $(P, Q, B)$-block map. (2) If $T$ doubly preserves any one $T R t$, then we have that $T$ is also a $(P, Q, B)$-block map.

(1) First case $s=(t+1)$ : Then $T$ preserves TR 1 by Lemma 7 .

Second case $s=(t+2)$ : Lemma 9 implies that $T$ preserves TR $(t+1)$. Hence $T$ preserves TR 1 by Lemma 7.

Third case $s \geq(t+3)$ : Then by Lemma $8, T$ preserves TR 1 .

Consequently, $T$ preserves TR 1 by the above three cases. Hence, $T$ doubly preserves TR 1 by Lemma 3. By Theorem $1, T$ is a $(P, Q, B)$-block map.

(2) By Lemma 10, $T$ preserves TR 1. By Lemma 3, $T$ doubly preserves TR 1 . Thus $T$ becomes a $(P, Q, B)$-block map by Theorem 1 .

Now we have the main theorem :

Theorem 2. The following are equivalent for $T$ :

1. T preserves any two $T R$ t and $T R s$, with $t<s$ and $(t+1)<p$;

2. $T$ doubly preserves any one $T R t$, with $1 \leq t \leq p$;

3. T preserves TR;

4. T is the $(P, Q, B)$-block map.

It holds trivally that 3 implies 1 and 3 implies 2. Moreover, by Lemma 2, we have that 4 implies the other items 1,2 and 3.

To show that 1 implies 4 , suppose that $T$ preserves TR $t$ and TR $s$, with $1 \leq t<s \leq p \leq q$. Then, by Lemma $11(1), T$ is the $(P, Q, B)$-block map.

To show that 2 implies 4 , suppose that $T$ doubly preserves TR $t$. Then $T$ is the $(P, Q, B)$-block map Lemma 11 (2).

Thus we obtained characterizations of the linear maps that preserve any two term rank between different matrix spaces.

\section{Conclusions}

There are many research articles on the linear operators which preserve term rank over some matrix spaces. However, there are few articles for the characterizations of the linear maps that preserve term rank between different matrix spaces over semirings. In this paper, we have characterized the linear maps which preserve term rank between different matrix spaces over anti-negative commutative semirings, which extend the previous results on characterizations of linear operators between the same matrix spaces. That is, a linear map $T$ from $p \times q$ matrix spaces into $m \times n$ matrix spaces preserves any two term ranks if and only if $T$ preserves all term ranks if and only if $T$ is a $(P, Q, B)$-block map. In the future, we may apply these results and this proof method to investigate the linear preserver problems over various semirings. We hope to apply these results to characterize the linear maps that preserve the semiring rank between different matrix spaces, which extends the previous results on characterizations of linear operators that preserve the semiring rank between the same matrix spaces.

Author Contributions: Create and conceptualize ideas, K.T.K. and S.-Z.S.; writing-original draft preparation, K.T.K. and S.-Z.S.; writing-review and editing, Y.B.J.; funding acquisition, S.-Z.S. All authors have read and agreed to the published version of the manuscript. 
Funding: This research was supported by Basic Science Research Program to RIBS of Jeju National University through the National Research Foundation of Korea (NRF) funded by the Ministry of Education (No. 2019R1A6A1A10072987).

Conflicts of Interest: The authors declare no conflict of interest.

\section{References}

1. Beasley, L.B.; Pullman, N.J. Term-rank, permanent, and rook-polynomial preservers. Linear Algebra Appl. 1987, 90, 33-46. [CrossRef]

2. Kang, K.T.; Song, S.Z.; Beasley, L.B. Linear preservers of term ranks of matrices over semirings. Linear Algebra Appl. 2012, 436, 1850-1862. [CrossRef]

3. Song, S.Z.; Beasley, L.B. Linear transformations that preserve term rank between different matrix spaces. J. Korean Math. Soc. 2013, 50, 127-136. [CrossRef]

4. Beasley, L.B. Preservers of term ranks and star cover numbers of symmetric matrices. Electron. J. Linear Algebra 2016, 31, 549-564. [CrossRef]

5. Beasley, L.B.; Song, S.Z.; Kang, K.T. Preservers of term ranks of symmetric matrices. Linear Algebra Appl. 2012, 436, 1727-1738. [CrossRef]

6. Beasley, L.B.; Song, S.Z. Linear operators that preserve term ranks of matrices over semirings. Bull. Malays. Math. Sci. Soc. 2014, 37, 719-725.

7. Kang, K.T.; Song, S.Z.; Jun, Y.B. Linear operators that strongly preserve regularity of fuzzy matrices. Math. Commun. 2010, 15, 243-254.

(C) 2020 by the authors. Licensee MDPI, Basel, Switzerland. This article is an open access article distributed under the terms and conditions of the Creative Commons Attribution (CC BY) license (http:/ / creativecommons.org/licenses/by/4.0/). 